\title{
Partida de um reator UASB na codigestão de água negra com resíduos orgânicos alimentares
}

\section{Start-up of a UASB reactor for codigestion of blackwater and kitchen waste}

\author{
Data de entrada: \\ 25/09/2019 \\ Data de aprovação: \\ $17 / 08 / 2020$
}

Larissa de Oliveira Prado ${ }^{1 *}$ | Gabriel Magalhães Chiquito' | Paula Loureiro Paulo | Marc Árpád Boncz'

DOI: https://doi.org/10.36659/dae.2022.011

ORCID ID

Prado LO (D) https://orcid.org/0000-0001-9303-7862

Chiquito GM (iD https://orcid.org/0000-0002-2873-8395
Paulo PL (ID) https://orcid.org/0000-0002-8091-6486 Boncz MA (ID) https://orcid.org/0000-0001-7618-8685

\section{Resumo}

Novas concepções para o tratamento de fontes segregadas do esgoto doméstico têm sido propostas como alternativa ao tratamento convencional. Assim, com o objetivo de investigar o desempenho de um reator UASB, durante a codigestão de água negra com resíduos orgânicos alimentares (AN-ROA), quanto à cinética de degradação da matéria orgânica e a conservação de nutrientes para reúso do efluente, foi realizada a partida do reator em bateladas com períodos de 12 e 6 dias. Durante esses períodos, o reator pôde alcançar satisfatoriamente a biodegradação da AN-ROA, com remoção de $86 \%$ e $84 \%$ de DQQ total (DQO $\mathrm{T}_{\mathrm{T}}$ ), respectivamente, sendo a maior parte consumida na forma de sólidos em suspensão $\left(\mathrm{DQO}_{\mathrm{SS}}\right)$. Durante a partida, o reator apresentou aumento da alcalinidade a bicarbonato $\left(\mathrm{HCO}_{3}^{-}\right)$e manteve $\mathrm{pH}$ em faixa favorável a digestão anaeróbia, sem acúmulo de ácidos graxos voláteis (AGV). Os nutrientes fosfato $\left(\mathrm{PO}_{4}{ }^{3-}\right)$ e nitrogênio total (NT) foram conservados no efluente tratado.

Palavras-chave: Água negra. Codigestão. Cinética. Resíduos orgânicos alimentares. tratamento descentralizado.

\section{Abstract}

New concepts for treatment of source-separated household wastewater have been proposed as an alternative for traditional treatment. Thus, with the objective of investigating the performance of a UASB reactor, codigestion of blackwater and kitchen waste (BW-KW) for organic matter degradation and nutrient conservation in the effluent (for reuse) was realized; start-up of the reactor was performed in batch mode with periods of 12 and 6 days. During these periods, the reactor was able to satisfactorily achieve biodegradation of the BW-KW mixture, with $86 \%$ and $84 \%$ removal of total COD (COD)), respectively, the major part of which was consumed in the form of suspended solids $\left(\mathrm{COD}_{s S}\right)$. During the start-up, the reactor shows an increase in bicarbonate alkalinity $\left(\mathrm{HCO}_{3}^{-}\right)$and maintains $a \mathrm{pH}$ in the range favorable to anaerobic digestion, without accumulation of volatile fatty acids (VFA). Nutrients phosphate $\left(\mathrm{PO}_{4}{ }^{3-}\right)$ and total nitrogen (NT) were shown to be conserved in the treated effluent.

Keywords: Blackwater. Codigestion. Decentralized treatment. Kinetics. Kitchen waste.

\footnotetext{
1 Universidade Federal de Mato Grosso do Sul (UFMS) - Campo Grande - Mato Grosso do Sul - Brasil.

* Autora correspondente: lariprado27agmail.com.
} 


\section{INTRODUÇÃO}

Os atuais sistemas consolidados de saneamento têm como característica o tratamento centralizado de efluentes domésticos com pouca, ou nenhuma, recuperação de recursos para reutilização. Por isso, novas concepções para o tratamento descentralizado, de fontes segregadas do esgoto doméstico, vêm sendo propostas como alternativas ao sistema convencional, assim como o tratamento de água negra (fezes, urina e água), que pode tanto ser tratada separadamente (DE GRAAFF et al., 2010; PAULO et al., 2013; TODT e JENSSEN, 2015; OARGA-MULEC et al., 2017 e JIN et al., 2018) como em codigestão com resíduos orgânicos alimentares (KUJAWA-ROELEVELD et al., 2003; KUJAWA ROELEVELD et al., 2005; ELMITWALLI et al., 2006; LUOSTARINEN; RINTALA, 2007 e WENDLAND et al., 2007), obtendo eficientes resultados na produção de biogás e na recuperação de nutrientes. Além disso, o tratamento descentralizado de fonte segregada de água negra e de resíduos sólidos orgânicos, como parte de um sistema de saneamento ecológico, não só promove a recuperação de recursos como também visa a uma menor contaminação de outros efluentes, como a água cinza (normalmente livre de matéria fecal e microorganismos patogênicos), e do meio ambiente. Outro fator a ser considerado é a maior simplicidade dos sistemas que podem ser utilizados como tratamento domiciliar ou comunitário local, sendo úteis em áreas rurais devido às circunstâncias especiais, como a baixa densidade populacional e, especialmente em países subdesenvolvidos ou em desenvolvimento, devido à sua facilidade de uso e custo (LUOSTARINEN et al., 2007).

A segregação de materiais como fezes, resíduos orgânicos alimentares e urina permite tratá-los como fontes de recursos que podem ser recuperados, visto que os dois primeiros contêm grande quantidade de matéria orgânica, adequada para compostagem e condicionamento do solo, en- quanto a urina contém a maior fração de nitrogênio e fósforo (WIELEMAKER, WEIJMA e ZEEMAN, 2018). Assim, o tratamento via digestão anaeróbia que emprega ações complexas de microbiologia anaeróbia e ações físico-químicas, para decompor, estabilizar e separar os materiais fecais apresenta uma maneira regular de tratar água negra e, com o uso de reator, obter maior eficiência na recuperação de bioenergia (HU et al., 2016) e nutrientes. À vista disso, pesquisas sobre a codigestão de água negra com resíduos sólidos orgânicos foram realizadas sobre diferentes aspectos, assim como: diferentes temperaturas, tempo de detenção hidráulica (TDH), concentração de alimentação e diferentes tipos de reatores (tanque séptico, reator UASB de uma ou duas fases, reator de mistura completa (CSTR) e sistema de acumulação (AC)), obtendo resultados positivos no desempenho estavél dos sistemas, nas remoções de sólidos em suspensão e DQQ total, além da produção de biogás (KUJAWA-ROELEVELD et al., 2003; KUJAWA-ROELEVELD et al., 2005; ELMITWALLI et al., 2006; LUOSTARINEN; RINTALA, 2007 e WENDLAND et al., 2007). Assim, de forma a obter maior compreensão sobre a eficiência da codigestão de água negra e resíduos orgânicos alimentares (AN-ROA), a presente pesquisa tem como objetivo avaliar o desempenho da partida e operação de um reator UASB, na biodegradação da AN-ROA, com base na cinética de degradação da matéria orgânica e a conservação de nutrientes para reúso do efluente.

\section{MATERIAL E MÉTODOS}

\subsection{Reator UASB e resíduos para o tratamento}

Um reator UASB construído em PVC, com volume de trabalho de $112 \mathrm{~L}$, diâmetro de $200 \mathrm{~mm}$, altura de 2,7 $\mathrm{m}$ (com 5 amostradores da base ao topo) e separador trifásico em forma de braço lateral em $45^{\circ}$, foi utilizado para codigestão anaeróbia de água negra (AN) e resíduos orgânicos alimen- 
tares (ROA). 0 reator foi inoculado com $37 \mathrm{~L}$ de biomassa metanogênica (95 gSV.L-1) originada de reator UASB tratando efluentes da fábrica local de engarrafamento de refrigerante.

A AN, coletada em banheiro experimental com volume de descarga convencional, e os ROA foram triturados antes de serem armazenados em um tanque de equalização com volume controlável, e a mistura AN-ROA era, então, bombeada ao reator. Os ROA foram adicionados proporcionalmente ao volume de AN adotado, que foi de 130 $\mathrm{L}$, sendo a quantidade de ROA adicionada de 1,89 $\mathrm{kg}$, valores que representam aproximadamente a produção diária em uma residência de 4 pessoas, considerando uma geração média de esgoto domestico de $130 \mathrm{~L}$ per capita por dia (ABNT, NBR 7229/1993), sendo 75\% água cinza (HERNÁNDEZ LEAL et al., 2011), e, portanto, a geração de AN de 32,5 L per capita por dia; e considerando a massa coletada de resíduos domésticos e públicos de 0,92 kg/hab./dia (BRASIL, 2018), sendo a fração orgânica de 51,4\% (IPEA, 2012). Durante a alimentação do reator, os ROA eram adicionados ao tanque de equalização em porções dívidas, sendo 4 vezes quando o volume do tanque de equalização era de $25 \mathrm{~L}$ e 2 vezes quando o volume no tanque era de $50 \mathrm{~L}$. A Fig. 1 ilustra o caminho das AN-ROA até o reator UASB.

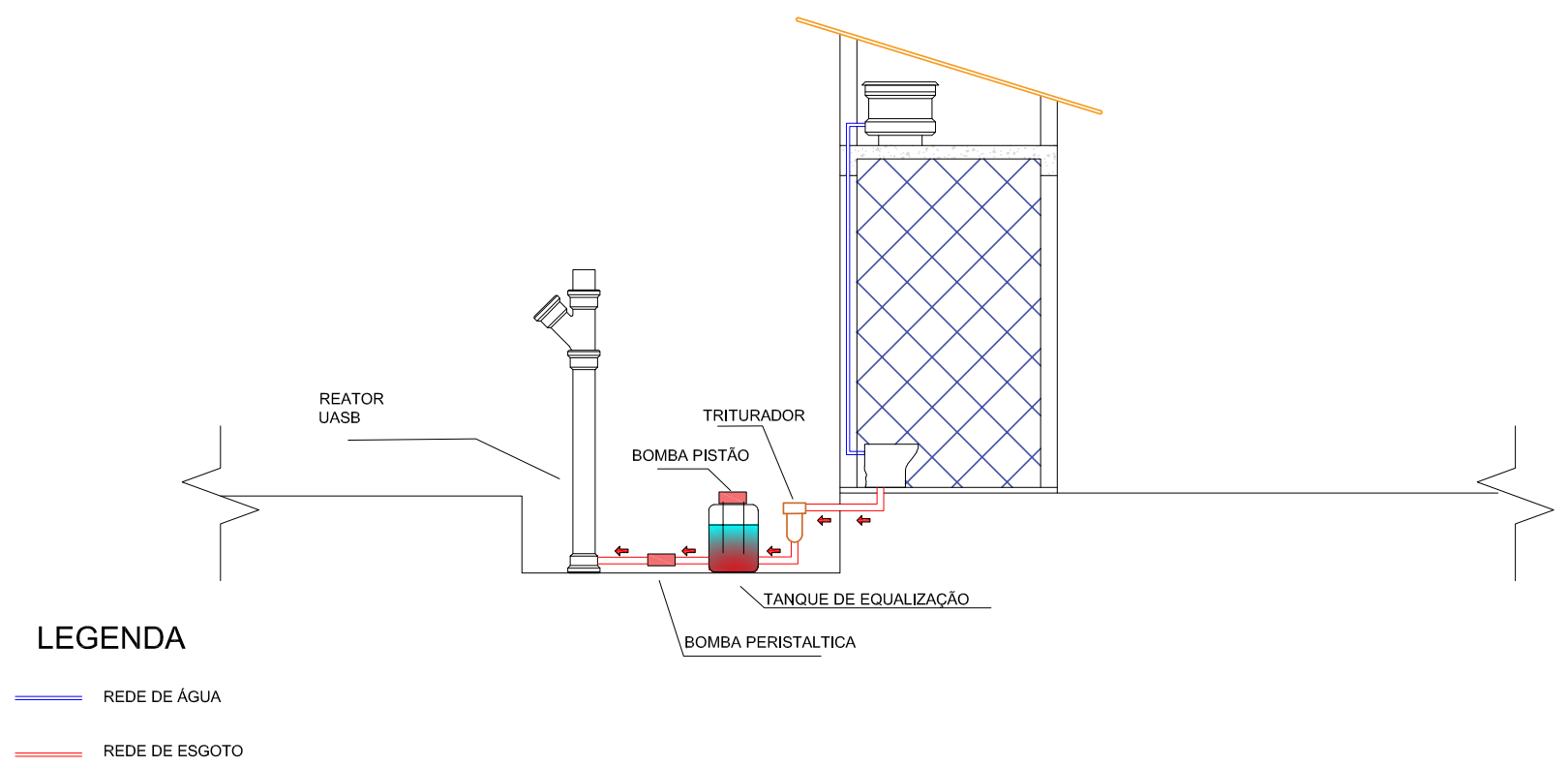

Figura 1 - Sistema da codigestão de AN e ROA.

\subsection{Teste da Atividade Metanogênica Especifica (AME)}

Para verificar a AME da biomassa de inóculo no reator foi realizado um ensaio de bancada, utilizando acetato como substrato, preparado de acordo com Chernicharo (2007). O ensaio foi realizado em triplicata, utilizando frascos âmbar com volume de $620 \mathrm{~mL}$ ( $500 \mathrm{~mL}$ de líquido e $120 \mathrm{~mL}$ de headspace). A biomassa utilizada (2 gSV.L-1 para cada frasco), proveniente de um reator UASB, tratando efluentes da fábrica local de engarrafamento de refrigerante, obedeceu à relação de 1:1,4 (1 gD$\mathrm{QO}_{\text {substrato }} / 1,4 \mathrm{gSV}_{\text {biomassa }}$ ). $\mathrm{O}$ gás nitrogênio puro foi utilizado no headspace de todos os frascos que, após serem selados, foram incubados a $30 \pm 3^{\circ} \mathrm{C}$, sob agitação mecânica (75 rpm), para promover condições anaeróbias. 
A formação do biogás foi medida, em todos os frascos, usando o método de deslocamento (com $\mathrm{NaOH}$ a $18 \%$ para absorver o $\mathrm{CO}_{2}$ do biogás). A primeira medição foi realizada após um dia de experimento, sendo as demais realizadas de acordo com a produção de biogás. Após cada medição, todos os frascos eram homogeneizados, e depois de aguardar um minuto para que a biomassa sedimentasse, retirava-se por meio de uma seringa $10 \mathrm{ml}$ de amostra para determinação da $\mathrm{DQO}_{\mathrm{T}}$ (total) e pH.

\subsection{Partida e operação}

A biomassa foi inoculada a partir da base do reator, ocupando 1/3 do volume de trabalho do mesmo. Em seguida, as AN-ROA do tanque de equalização foram bombeadas para o reator a uma vazão

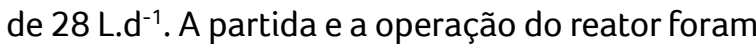
realizadas em bateladas de 12 dias (baseado em testes preliminares de AME) e 6 dias. Inicialmente, a partida teve duração de 81 dias, para que a biomassa se adaptasse à AN-ROA e aumentasse a velocidade de remoção da DQO. Nesse período, o reator operou 6 bateladas com períodos de 12 dias, com uma vazão de recirculação de $28{\mathrm{~L} . \mathrm{d}^{-1}}^{-1}$. Posteriormente, foram realizadas 2 bateladas, nas quais houve o aumento da carga orgânica volumétrica (COV), com diminuição do período de operação para 6 dias, por batelada, e maior contato do efluente com a manta de lodo, por meio do au-

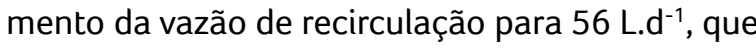
proporcionou o dobro da velocidade ascensional da qual o reator estava recebendo nas bateladas com período de 12 dias (Tabela 1).

Tabela 1 - Parâmetros de projeto.

\begin{tabular}{|c|c|c|c|}
\hline $\begin{array}{c}\text { Parâmetro de } \\
\text { Projeto }\end{array}$ & Unidade & $\begin{array}{c}\text { Bateladas de } \\
\mathbf{1 2} \text { dias }\end{array}$ & $\begin{array}{c}\text { Bateladas de } \\
\mathbf{6} \text { dias }\end{array}$ \\
\hline $\begin{array}{c}\text { Quantidade de } \\
\text { bateladas }\end{array}$ & - & 6 & 2 \\
\hline COV & $\mathrm{kgDQO} \cdot \mathrm{m}^{-3} \cdot \mathrm{d}^{-1}$ & 0,7 & 1,1 \\
\hline $\begin{array}{c}\text { Velocidade } \\
\text { ascensional }\end{array}$ & $\mathrm{cm} \cdot \mathrm{h}^{-1}$ & 3,7 & 7,4 \\
\hline
\end{tabular}

\subsection{Procedimentos analíticos}

Amostras de entrada e saída do reator foram coletadas a cada 4 e 2 dias, nas bateladas com períodos de 12 e 6 dias, respectivamente, para análises. Para o cálculo da média e desvio padrão, dos parâmetros de entrada no reator, era realizada análise das amostras de AN, antes da adição dos ROA, e da mistura AN-ROA, após a adição dos ROA, no tanque de equalização. Em seguida, a concentração da mistura AN-ROA, ao longo do dia, era estimada a partir do balanço de entrada e saída de água negra no tanque de equalização, por meio de uma planilha Excel.

Os parâmetros medidos foram: $\mathrm{pH}$, nitrogênio total (NT), nitrogênio amoniacal, DQQOT (total), DQOF (filtrada), carbono orgânico total (COT), sólidos totais (ST), sólidos voláteis (SV), alcalinidade a bicarbonato, sulfeto, sulfato, fosfato e ácido graxo volátil (AGV), determinados de acordo com o método correspondente, conforme descrito em Standard Methods for the Examination of Water and Wastewater (APHA, 2012), sendo as análises de DQO realizadas em duplicata. A concentração de DOQO de sólidos em suspensão (DQOSS) foi calculada como a diferença entre a DOOT e DQOOF ${ }_{F}$ A Tabela 2 apresenta a composição da mistura AN-ROA bruta, nas bateladas com período de 12 e 6 dias.

Tabela 2 - Valores médios estimados referentes à composição de AN-ROA bruta para o tratamento em batelada pelos períodos de 12 dias e de 6 dias.

\begin{tabular}{|c|c|c|c|}
\hline Parâmetro & Unidade & $\begin{array}{c}\text { Bateladas de 12 dias } \\
\text { Entrada }\end{array}$ & $\begin{array}{c}\text { Bateladas de 6 dias } \\
\text { Entrada }\end{array}$ \\
\hline DQO $_{\top}$ & $\mathrm{g} \cdot \mathrm{L}^{-1}$ & $2,64 \pm 0,91$ & $2,25 \pm 0,40$ \\
\hline DQO $_{\mathrm{SS}}$ & $\mathrm{g} \cdot \mathrm{L}^{-1}$ & $1,52 \pm 0,76$ & $1,26 \pm 0,54$ \\
\hline DQO $_{\mathrm{F}}$ & $\mathrm{g} \cdot \mathrm{L}^{-1}$ & $1,12 \pm 0,34$ & $0,99 \pm 0,14$ \\
\hline $\mathrm{ST}$ & $\mathrm{g} \cdot \mathrm{L}^{-1}$ & $2,28 \pm 0,72$ & $1,61 \pm 0,21$ \\
\hline $\mathrm{SV}$ & $\mathrm{g} \cdot \mathrm{L}^{-1}$ & $1,10 \pm 0,59$ & $0,75 \pm 0,25$ \\
\hline $\mathrm{pH}$ & & $7,6 \pm 0,5$ & $7,5 \pm 0,5$ \\
\hline Alc & $\mathrm{g} \cdot \mathrm{L}^{-1}$ & $0,97 \pm 0,28$ & $0,69 \pm 0,20$ \\
\hline Alc & $\mathrm{g} \cdot \mathrm{L}^{-1}$ & $1,95 \pm 0,86$ & $1,32 \pm 0,22$ \\
\hline Sulfato & $\mathrm{mg} \cdot \mathrm{L}^{-1}$ & $15,3 \pm 5,5$ & $25,6 \pm 5,0$ \\
\hline Sulfeto & $\mathrm{mg} \cdot \mathrm{L}^{-1}$ & $24,9 \pm 20,8$ & $33,7 \pm 3,9$ \\
\hline $\mathrm{NT}$ & $\mathrm{mg} \cdot \mathrm{L}^{-1}$ & $289 \pm 48$ & $322 \pm 22$ \\
\hline Amônio & $\mathrm{mg} \cdot \mathrm{L}^{-1}$ & $202 \pm 43$ & $186 \pm 77$ \\
\hline Fosfato & $\mathrm{mg} \cdot \mathrm{L}^{-1}$ & $113,76 \pm 70,57$ & $112,48 \pm 57,62$ \\
\hline COT & $\mathrm{mg} \cdot \mathrm{L}^{-1}$ & $619,73 \pm 137,38$ & $464,53 \pm 3,18$ \\
\hline C:N & & $2,14 \pm 0,22$ & $1,45 \pm 0,09$ \\
\hline AGV & $\mathrm{mg} \cdot \mathrm{L}^{-1}$ & $370,84 \pm 78,64$ & $216,36 \pm 1,95$ \\
\hline
\end{tabular}


O modelo de primeira ordem (Eq. 1) foi utilizado para determinar os parâmetros cinéticos com os resultados da DQOT.

$C=C_{0} e^{-k t}$

Onde C: concentração de $\mathrm{DQO}_{\mathrm{T}}\left(\mathrm{mg} \cdot \mathrm{L}^{-1}\right) ; \mathrm{CO}$ : concentração inicial de $\mathrm{DQO}_{\mathrm{T}}\left(\mathrm{mg} \cdot \mathrm{L}^{-1}\right)$; $\mathrm{k}$ : constante de reação $\left(d^{-1}\right)$; e t: tempo $(d)$.

\section{RESULTADOS E DISCUSSÃO}

\subsection{Atividade Metanogênica Especifica (AME)}

A AME da biomassa foi estudada em ensaio de bancada com foco no consumo de substrato e produção de gás metano. Como é possível visualizar na Fig. 2, a produção de gás metano começou imediatamente após o início do experimento, porém a velocidade máxima da conversão da matéria orgânica a gás metano só foi alcançada após aproximadamente 3 dias.

Em aproximadamente 14 dias, após inicio do ensaio, foram alcançados $93 \%$ de remoção de DOOT (DQO total inicial $=1,38 \mathrm{~g} . \mathrm{L}^{-1}$ ) e uma pro-

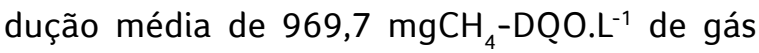
metano (média de 70\% de conversão de DQQO em $\mathrm{CH} 4$ ), com AME de 0,062 $\mathrm{gCH}_{4}$-DQQ O.gSV.d ${ }^{-1}$ (Fig. 2), o que sugere que possivelmente parte da DQO foi convertida em biomassa.
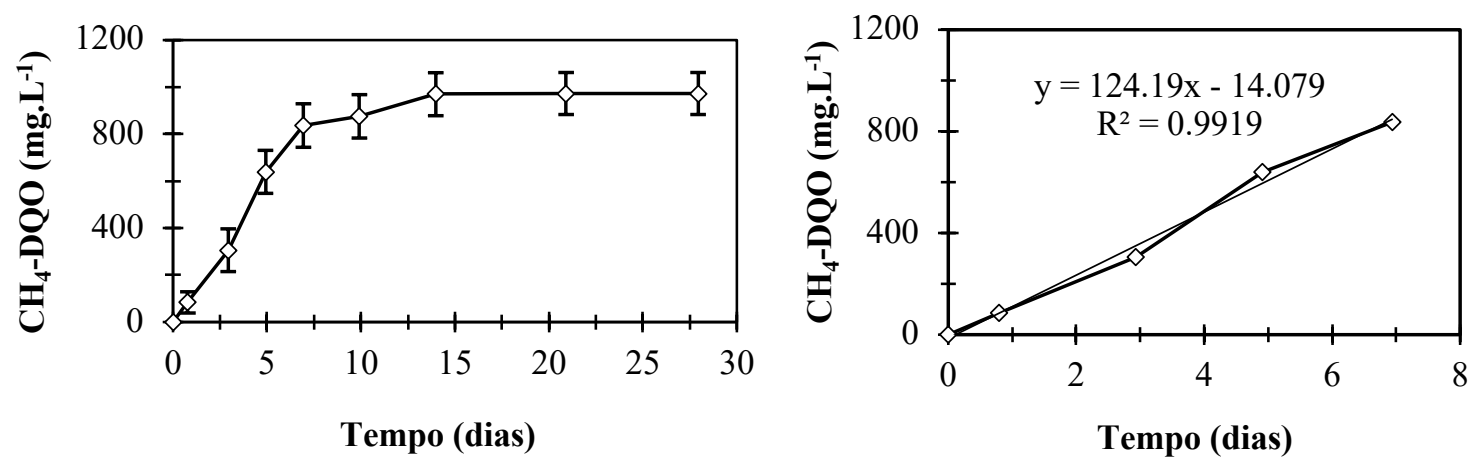

Figura 2 - Produção acumulada de biogás do teste da AME.

\subsection{Partida e Desempenho do Reator UASB}

A partida do reator UASB ocorreu em bateladas com períodos de 12 e 6 dias, a Tabela 3 apresenta a composição da saída de AN-ROA trata$\mathrm{da}$, em ambos os períodos. Nas bateladas com período de 12 dias, a eficiência de remoção de sólidos totais (ST) foi de $56 \%$, durante o tratamento, sendo a remoção mínima de $34 \%$ e a máxima de $75 \%$. Com o aumento da velocidade ascensional, nas bateladas com período de 6 dias, houve aumento do arraste de partículas sólidas na AN-ROA tratada, diminuindo a eficiência de remoção de ST para $43 \%$.
Tabela 3 - Média dos valores referentes à AN-ROA tratada para o período de 12 dias e de 6 dias.

\begin{tabular}{|c|c|c|c|}
\hline Parâmetro & Unidade & $\begin{array}{c}\text { Bateladas de } 12 \text { dias } \\
\text { Saída }\end{array}$ & $\begin{array}{c}\text { Bateladas de } 6 \text { dias } \\
\text { Saída }\end{array}$ \\
\hline $\mathrm{DQO}_{\mathrm{T}}$ & $g \cdot L^{-1}$ & $0,36 \pm 0,19$ & $0,36 \pm 0,11$ \\
\hline $\mathrm{DQO}_{\mathrm{SS}}$ & $g \cdot L^{-1}$ & $0,22 \pm 0,16$ & $0,09 \pm 0,09$ \\
\hline $\mathrm{DQO}_{\mathrm{F}}$ & $g \cdot \mathrm{L}^{-1}$ & $0,14 \pm 0,06$ & $0,27 \pm 0,02$ \\
\hline ST & $g \cdot \mathrm{L}^{-1}$ & $1,01 \pm 0,09$ & $0,92 \pm 0,08$ \\
\hline SV & $g \cdot \mathrm{L}^{-1}$ & $0,33 \pm 0,11$ & $0,29 \pm 0,04$ \\
\hline $\mathrm{pH}$ & & $7,7 \pm 0,3$ & $7,7 \pm 0,4$ \\
\hline Alc $_{p}$ & $g \cdot \mathrm{L}^{-1}$ & $1,42 \pm 0,20$ & $1,51 \pm 0,31$ \\
\hline $\mathrm{Alc}_{\mathrm{T}}$ & $\mathrm{g} \cdot \mathrm{L}^{-1}$ & $2,27 \pm 0,56$ & $2,58 \pm 0,14$ \\
\hline Sulfato & $\mathrm{mg} \cdot \mathrm{L}^{-1}$ & $8,7 \pm 3,4$ & $16,1 \pm 0,1$ \\
\hline Sulfeto & $\mathrm{mg} \cdot \mathrm{L}^{-1}$ & $6,4 \pm 8,7$ & $15,06 \pm 4,33$ \\
\hline NT & $\mathrm{mg} \cdot \mathrm{L}^{-1}$ & $212,53 \pm 44,92$ & $187,85 \pm 1,04$ \\
\hline Amônio & $\mathrm{mg} \cdot \mathrm{L}^{-1}$ & $0,90 \pm 0,34$ & $0,70 \pm 0,00$ \\
\hline Fosfato & $\mathrm{mg} \cdot \mathrm{L}^{-1}$ & $183,89 \pm 71,96$ & $153,00 \pm 33,04$ \\
\hline COT & $\mathrm{mg} \cdot \mathrm{L}^{-1}$ & $619,73 \pm 137,38$ & $464,53 \pm 3,18$ \\
\hline $\mathrm{C}: \mathrm{N}$ & & $2,14 \pm 0,22$ & $1,45 \pm 0,09$ \\
\hline AGV & $\mathrm{mg} \cdot \mathrm{L}^{-1}$ & $370,84 \pm 78,64$ & $216,36 \pm 1,95$ \\
\hline
\end{tabular}


Ao final das bateladas, os dois períodos (12 dias e 6 dias) apresentaram resultados semelhantes para os valores de ST, SV e o percentual de matéria orgânica, sendo a razão SV/ST final de 0,3. Assim, acredita-se que nas bateladas com período de 12 dias ocorreu maior sedimentação de material particulado, visto a maior concentração de ST inicial e menor velocidade ascensional. Além disso, a atividade metabólica do lodo também pode ter contribuído para remoção dos sólidos, da fração orgânica, melhorando o processo da digestão anaeróbia e permitindo a diminuição do período das bateladas, sem comprometer o tratamento. A Fig. 3 apresenta a remoção de ST e SV nas bateladas com período de 12 e 6 dias, sendo os ST a soma dos Sólidos fixos (SF) e SV.
A

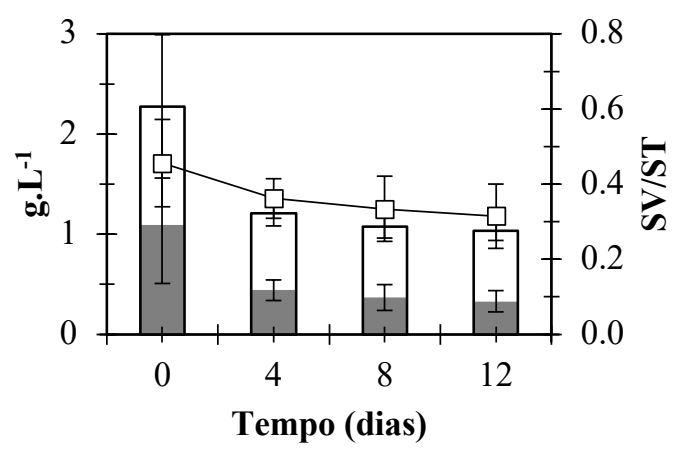

B

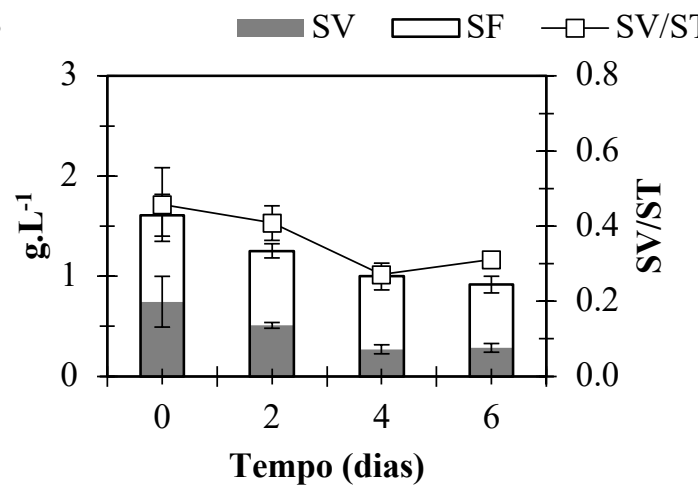

Figura 3 - Remoção de Sólidos ao longo das bateladas, sendo A - remoção obtida no tratamento com bateladas de período de 12 dias e B - remoção obtida no tratamento com bateladas de período de 6 dias.

Em pesquisas já realizadas com a codigestão de água negra, bons resultados foram obtidos com TDHs a partir de 5 dias. Em uma pesquisa de Wendland et al. (2007), estudando a codigestão de água negra com resíduos orgânicos alimentares em reator de mistura completa (CSTR), ao estudarem os TDHs de 10, 15 e 20 dias, os autores afirmam que, mesmo em TDH de 10 dias, a digestão estável de água negra com resíduos orgânicos alimentares pôde obter $50 \%$ de remoção de DQO. Luostarinen e Rintala (2007), estudando também a codigestão de água negra com resíduos orgânicos alimentares, mas em reator UASB de duas fases, com valores de TDHs mais baixos $(3,4$ $+1,3$ dias e 3,4 + 1,4 dias, para codigestão a 10 e $20^{\circ} \mathrm{C}$, respectivamente), puderam concluir que os reatores foram eficientes na remoção de material orgânico sólido e dissolvido, removendo mais de 95\% de sólidos em suspensão e $90 \%$ de DQQO. Em uma pesquisa realizada por Kujawa-Roeleveld et al. (2005), a codigestão de água negra com resíduos orgânicos alimentares foi estudada a longo TDH, de 29 dias, o qual os autores acreditam ter contribuído para a redução de $E$. coli em aproximadamente 3 logs, além da redução da DOQ em $82 \%$. Já na pesquisa realizada por de Graaff et al. (2010), ao estudar o tratamento apenas da água negra concentrada, os autores relataram que o tratamento foi realizado com sucesso $(78 \%$ de remoção de DQO) em curto $\mathrm{TDH}$, sendo este de 8,7 dias. Desta forma, ao avaliar a codigestão de AN-ROA, na atual pesquisa, concluímos que o reator mostrou desempenho satisfatório e tratamento eficiente com períodos de tratamento comparáveis aos TDHs encontrados na literatura ou até curtos (12 e 6 dias). Além disso, a remoção de DOO obteve desempenho semelhante a testes preliminares de bancada, no qual a remoção de $85 \%$ da $\mathrm{DQO}_{\mathrm{T}}$ foi alcançada em um período de 12,9 dias, com remoção > 70\% em 3,7 dias. Já no 
reator UASB, o tempo necessário para a remoção de $86 \%$ de $\mathrm{DQO}_{\top}$ foi de 12 dias e, em consequência da retenção de sólidos e da adaptação da biomassa, pôde ser otimizado, após 81 dias de tratamento, passando o período das bateladas para 6 dias, sem prejuízo na remoção da $\mathrm{DQO}_{\mathrm{T}}(84 \%)$, demonstrando eficiente desempenho do reator durante a partida. Assim, acredita-se que a obtenção de $86 \%$ e $84 \%$ de remoção de $\mathrm{DQO}_{\mathrm{T}}$ seja um valor satisfatório que confirma a facilidade da degradação da água negra em codigestão com resíduos orgânicos alimentares, visto que no teste AME foi alcançada uma remoção de $93 \%$ de DQQO, em aproximadamente 14 dias, e o substrato utilizado (acetato) já estava pronto para o consumo das arqueias metanogênicas.
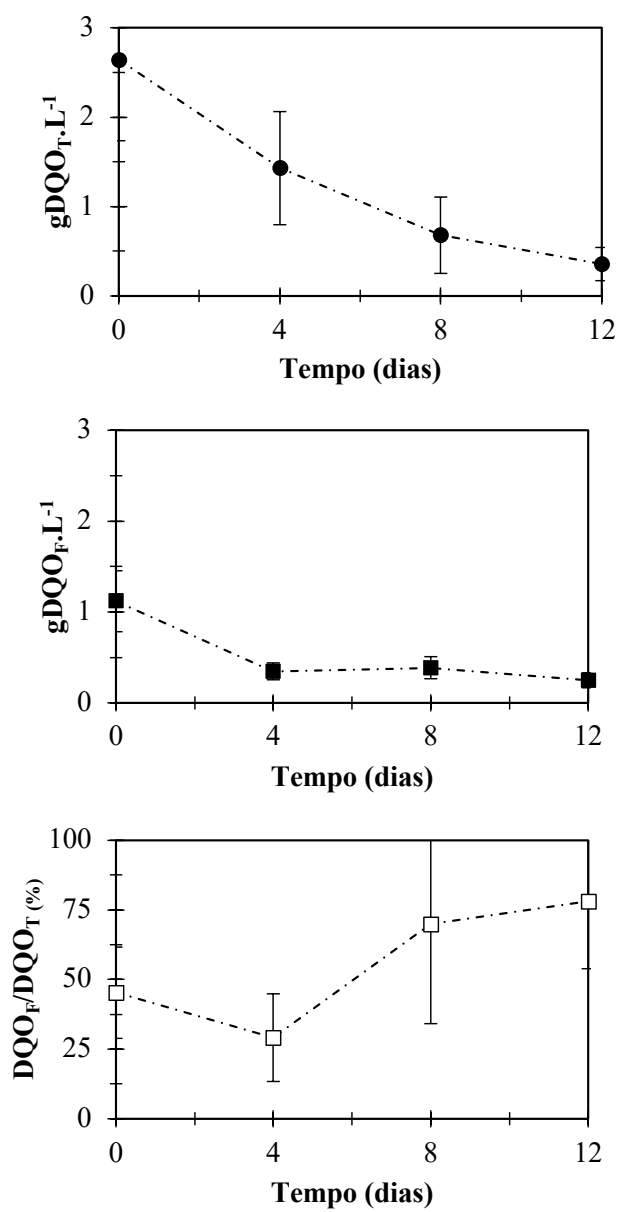

Durante toda a codigestão não foi possível medir a produção de biogás, assim acredita-se que o biogás possa ter escapado pelo braço lateral (saída do reator) ou por algum vazamento não identificado

A Fig. 4 apresenta os valores de DQO, ao longo das bateladas com período de 12 dias e seus percentuais de remoção. Nessas bateladas, a DQQOF, que representava a fração de DQ para ser biodegradada, correspondia a $42 \%$ da DQOT da AN-ROA bruta, e foi removida mais eficientemente em 4 dias. Após 8 dias houve aumento dos valores DQOF, possivelmente em consequência da formação de AGV, que foi removida no decorrer do tratamento, obtendo média de $87 \%$ de remoção de DQQOF no final das bateladas.
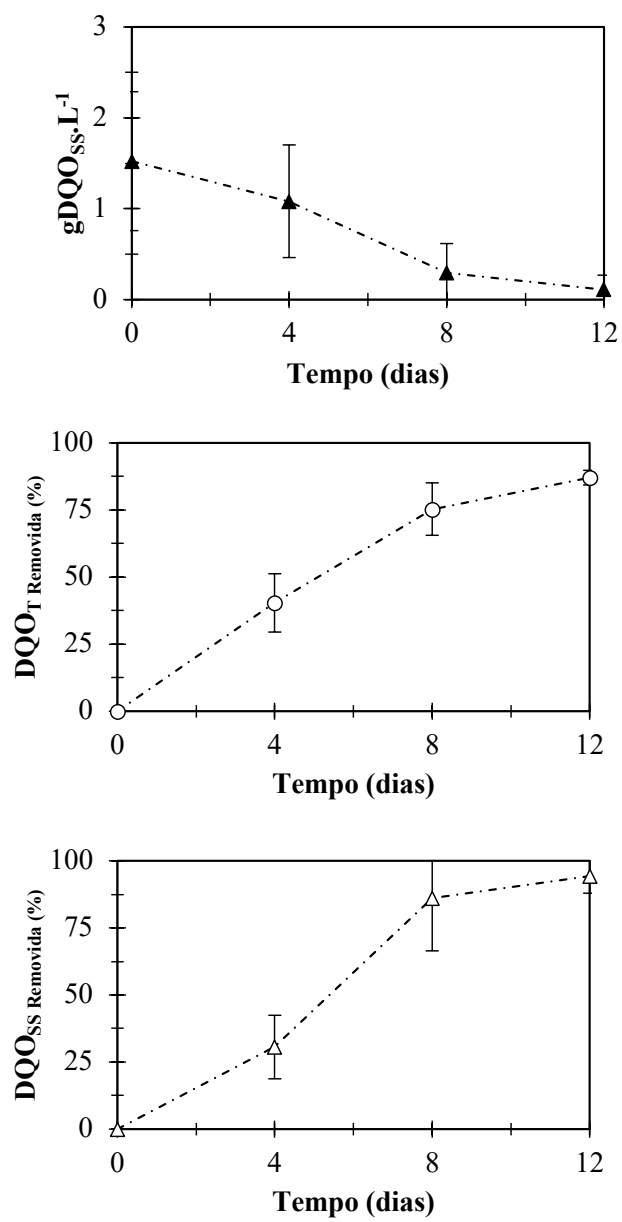

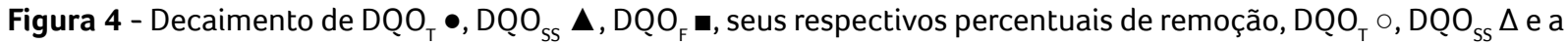
relação $\mathrm{DQO}_{\mathrm{F}} / \mathrm{DQO}_{\mathrm{T}} \square$, para o período de tratamento por 12 dias. 
No geral, para as bateladas com período de 12 dias, a maior parte da $\mathrm{DQO}_{\mathrm{T}}$ foi consumida na forma de $\mathrm{DQO}_{S \mathrm{~S}}$, com maior remoção entre 4 e 8 dias. Assim, supõe-se que a maior parte da DQO removida ficou acumulada no reator como material particulado, no qual uma parte contribuiu para aumento do leito de lodo e outra foi lentamente hidrolisada e convertida a biogás.

Como nas bateladas com período de 12 dias, nas bateladas com período de 6 dias a $\mathrm{DQO}_{\mathrm{F}}$ correspondia a $44 \%$ da $\mathrm{DQO}_{\mathrm{T}}$ da AN-ROA bruta, porém foi removida mais eficientemente em 2 dias (Fig. 5). Em 4 dias houve aumento de $\mathrm{DQO}_{\mathrm{F}}$, que foi removida no decorrer do tratamento, obten- do média de $73 \%$ de remoção de $\mathrm{DQO}_{\mathrm{F}}$ ao final das bateladas.

Também como nas bateladas com período de 12 dias, nas bateladas com período com 6 dias a maior parte da $\mathrm{DQO}_{\mathrm{T}}$ foi consumida na forma de $\mathrm{DQO}_{S S}$, sendo alcançada uma remoção de $93 \%$ de $\mathrm{DQO}_{\mathrm{sS}}$ ao final das bateladas, entretanto com consumo mais eficiente em dois períodos, em 2 e 6 dias (Fig. 5). Dessa forma, presume que, nesse caso também, a maior parte da DQO removida ficou acumulada no reator como material particulado, no qual uma parte também contribuiu para aumento do leito de lodo e outra foi lentamente hidrolisada e convertida em biogás.
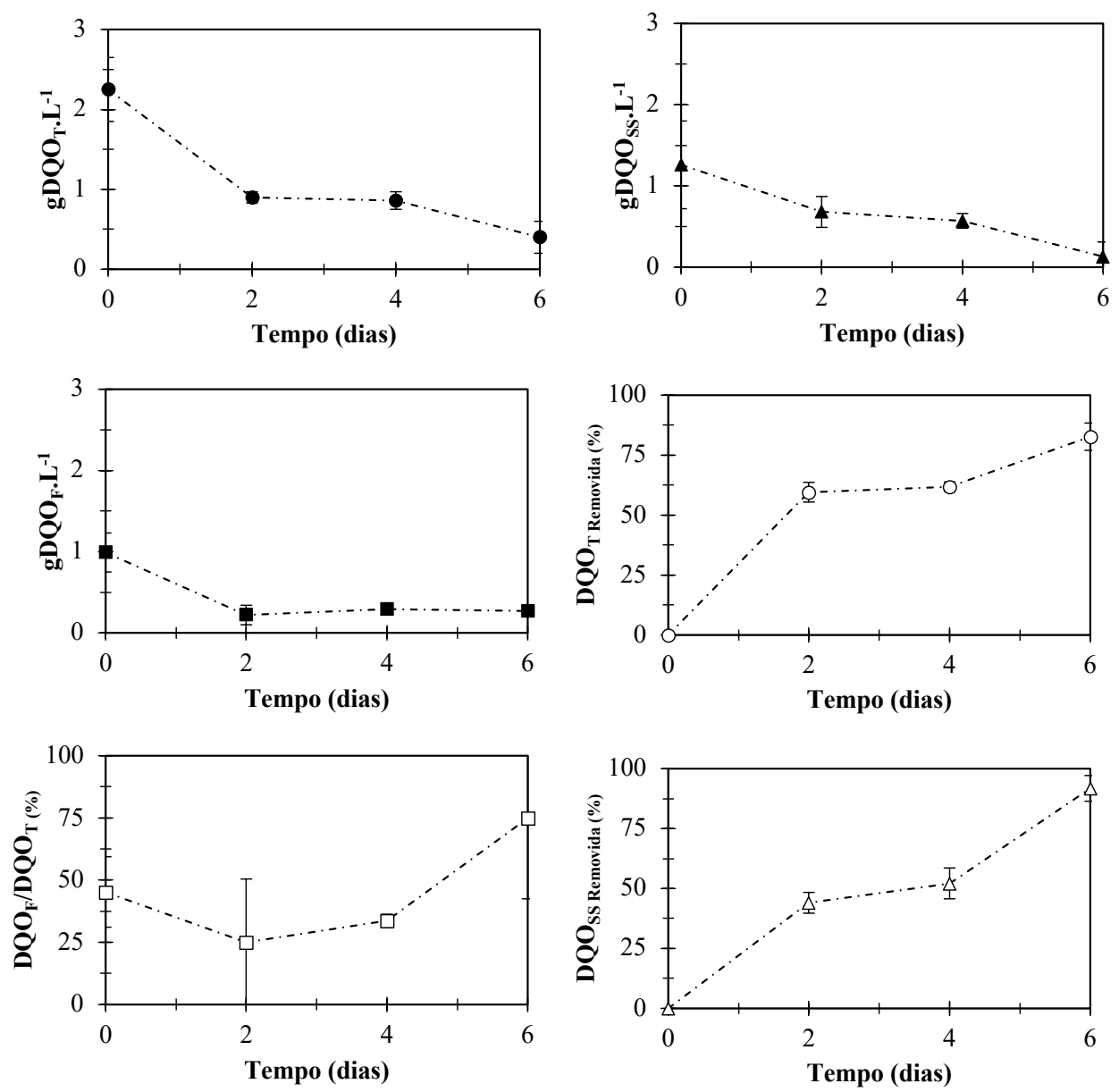

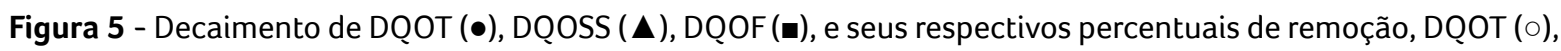
$\operatorname{DQOSS}(\Delta)$ e a relação DQOF/DQQOT ( $\square$ ), para o tratamento por período de 6 dias. 
Pesquisas já realizadas anteriormente também comprovaram a eficiência da codigestão de água negra e resíduos orgânicos alimentares na remoção de DQQ, sobretudo em relação ao material sedimentável. Luostarinen e Rintala (2007), ao estudarem a codigestão de água negra e os resíduos orgânicos alimentares em reator UASB de duas fases, obtiveram desempenho satisfatório, sendo a remoção de $\mathrm{DQO}_{\mathrm{T}} 88 \%-91 \%$ e de $\mathrm{DQO}_{\text {sS }}$ de $96 \%$ - 98\%. Também em estudos de codigestão de água negra e resíduos orgânicos alimentares, conduzidos por Kujawa-Roeleveld et al. (2005), a eficiência de remoção de DQ̣OT e DQOOSS foi de $82 \%$ e $94 \%$, respectivamente. Segundo os autores, a qualidade do efluente comprovou a capacidade do UASB de remover eficientemente materiais suspensos por meio da acumulação e da parcial conversão biológica.

Com o cálculo da cinética, de remoção da $\mathrm{DQO}_{\mathrm{T}}$, foi possível observar que, a partir da quinta batelada, a remoção de $\mathrm{DOO}_{\mathrm{T}}$ começou a obter eficiência $>80 \%$ em um período de 8 dias e aumento da velocidade de remoção (Fig. 6), considerando cinética de primeira ordem.

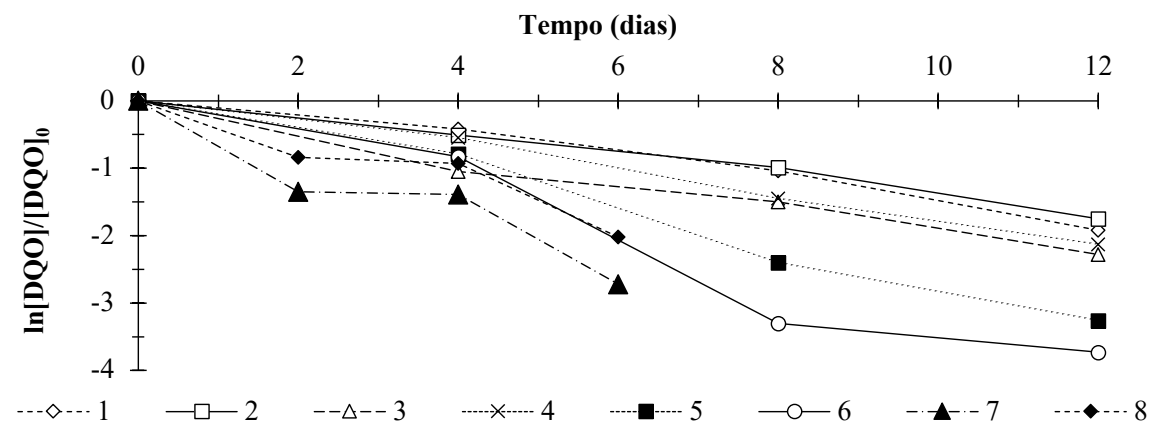

Figura 6 - Análise da degradação da DQOT, mostrando cinética de primeira ordem das bateladas, sendo de 1 a 6 as bateladas, em ordem cronológica, do período de 12 dias, e 7 e 8 as bateladas, em ordem cronológica, do período de 6 dias.

A Tabela 4 apresenta os valores das constantes de velocidade para cada batelada dos períodos de 12 e 6 dias, na qual é possível observar o au- mento da constante cinética, com o decorrer do tratamento, que possibilitou a redução do período das bateladas.

Tabela 4 - Constantes k de velocidade de remoção de DQOT para as bateladas, em ordem cronológica, dos tratamentos com período de 12 dias (1 a 6) e de 6 dias (7 e 8).

\begin{tabular}{|c|c|c|c|c|c|c|c|c|c|}
\hline Batelada & $\mathbf{1}$ & $\mathbf{2}$ & $\mathbf{3}$ & $\mathbf{4}$ & $\mathbf{5}$ & $\mathbf{6}$ & $\mathbf{8}$ \\
\hline Constante $k\left(\mathrm{~d}^{-1}\right)$ & 0,16 & 0,14 & 0,18 & 0,18 & 0,28 & 0,34 & 0,41 \\
\hline
\end{tabular}

Assim, mesmo com o aumento da COV, nas bateladas com período de 6 dias foram alcançados 84\% de remoção de $\mathrm{DQ}_{\mathrm{T}}$, que pôde ser associado a adaptação da biomassa. Além disso, a constante de velocidade de remoção de $\mathrm{DQO}_{\mathrm{T}}$ obteve valores de $k=0,41$ e 0,31 d 1, para primeira e segunda batelada com período de 6 dias. Acredita-se que a diferença das constantes de velocidade entre as duas bateladas possa ser consequência da concentração da DQOO de cada batelada ou das características da AN-ROA nesses períodos, como temperatura e a fração orgânica do material.

Além da remoção de material particulado, a remoção de COT também confirmou a eficiência 
da codigestão anaeróbia quanto à remoção de matéria orgânica da AN-ROA. A remoção da COT alcançada foi de $66 \%$ e $60 \%$, nas bateladas com períodos de 12 e 6 dias, respectivamente, assim como os resultados obtidos por Wendland et al. (2007), que também comprovaram o desempenho satisfatório da remoção de matéria orgânica, da codigestão anaeróbia de AN-ROA, em reator de mistura completa (CSTR), obtendo $52 \%$ de remoção de COT em TDH de 10 dias, 71\% em TDH de 15 dias e $72 \%$ em TDH de 20 dias.

\subsection{Estabilidade do reator e Nutrientes}

Nas bateladas com período de 12 dias, o reator apresentou aumento da alcalinidade a bicarbonato $\left(\mathrm{HCO}_{3}{ }^{-}\right)$do sistema e manteve o $\mathrm{pH}$ no reator em 7,60 \pm 0,06 (Fig. 7-A). O maior aumento de alcalinidade foi alcançado em 4 dias, provavelmente devido à formação de bicarbonato da conversão de acetato, uma vez que, em todas as bateladas, o maior consumo de DQOF também se estabeleceu no período de 4 dias, ou seja, período de maior consumo de acetato (Fig. 4). Além disso, nesse período também foi observado sutil aumento de amônio $\left(\mathrm{NH}_{4}^{+}\right)\left(227 \pm 72 \mathrm{mg} . \mathrm{L}^{-1}\right)$ que, assim como a formação de bicarbonato, pode decorrer das reações de hidrólise da uréia, formando amônia $\left(\mathrm{NH}_{3}\right)$ e $\mathrm{CO}_{2}$ que, quando combinados com a água, formam $\mathrm{NH}_{4}{ }^{+} \mathrm{e} \mathrm{HCO}_{3}{ }^{-}$, e, com isso, fornecem alcalinidade ao meio, assim como a teoria:

$\mathrm{H}_{2} \mathrm{~N}-\mathrm{CO}-\mathrm{NH}_{2}+\mathrm{H}_{2} \mathrm{O} \rightarrow 2 \mathrm{NH}_{3}+\mathrm{CO}_{2}$

$\mathrm{NH}_{3}+\mathrm{H}_{2} \mathrm{O}+\mathrm{CO}_{2} \rightarrow \mathrm{NH}_{4}^{+}+\mathrm{HCO}_{3}^{-}$

Desta forma, durante todas as bateladas, a concentração de AGV não afetou a operação do sistema que pode neutralizar a formação dos ácidos e tamponar o $\mathrm{pH}$ no reator.
A

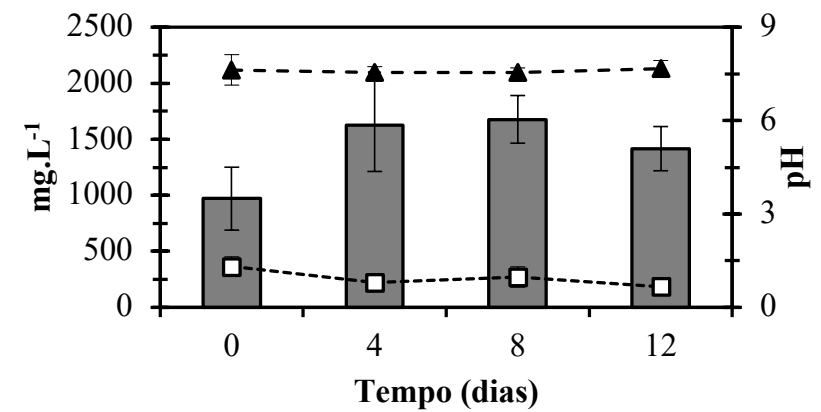

B

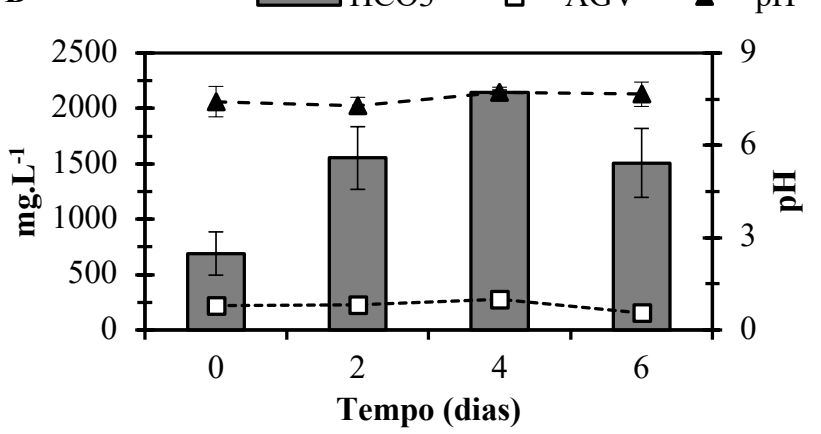

Figura 7 - Valores de alcalinidade a bicarbonato $\left(\mathrm{HCO}_{3}^{-}\right)$, $\mathrm{pH}$ e AGV, sendo $\mathrm{A}$ - tratamento em bateladas com períodos de 12 dias e $B$ - tratamento em bateladas com períodos 6 dias.

Nas bateladas com duração de 6 dias o reator também apresentou aumento da alcalinidade a bicarbonato, o que permitiu a estabilidade do $\mathrm{pH}$ em 7,54 \pm 0,19 (Fig. 7-B). No segundo dia das bateladas, houve consumo de $\mathrm{DQO}_{\mathrm{F}}$ (Fig. 5), que possivelmente contribuiu para o aumento da alcalinidade, devido à formação de bicarbonato da conversão de acetato. Ainda neste período, houve o aumento de $\mathrm{PO}_{4}{ }^{3-}\left(142,20 \pm 18,97 \mathrm{mg} \cdot \mathrm{L}^{-1}\right)$ e de amônio (233 $\left.\pm 60 \mathrm{mg} \cdot \mathrm{L}^{-1}\right)$ que, sutilmente, também podem ter contribuído para o aumento da alcalinidade. Durante todo o tratamento não foram observadas mudanças significativas, nos valores de AGV, que pudessem afetar a estabilidade do sistema.

No quarto dia, nas bateladas com período de 6 dias, houve aumentos da alcalinidade a bicarbo- 
nato $\left(\mathrm{HCO}_{3}{ }^{-}\right)$e do $\mathrm{PO}_{4}{ }^{3-}\left(172,67 \pm 26,35 \mathrm{mg} \cdot \mathrm{L}^{-1}\right)$ que foram atribuídos à fase de hidrólise de compostos orgânicos. Comportamento semelhante também foi relatado em pesquisa realizada por Cunha et al. (2018) no tratamento de água negra por reator UASB, no qual o aumento de $\mathrm{PO}_{4}{ }^{3-}$ - foi associado ao aumento da concentração de $\mathrm{HCO}_{3}{ }^{-}$. Na pesquisa, Cunha et al. (2018) investigaram os efeitos da relação $\mathrm{Ca}^{2+} / \mathrm{PO}_{4}{ }^{3-}$ na precipitação do $\mathrm{PO}_{4}{ }^{3-}$. Assim, quando os valores de $\mathrm{HCO}_{3}{ }^{-}$aumentaram de 1,2 a 2,7 g. $\mathrm{L}^{-1}$ houve decréscimo da concentração de $\mathrm{Ca}^{+}$solúvel e aumento no $\mathrm{pH}$, resultando no aumento nos valores de $\mathrm{PO}_{4}{ }^{3-}$, que foram relacionados à hidrólise de compostos contendo fósforo e/ ou o restabelecimento de $\mathrm{PO}_{4}{ }^{3-}$ do $\mathrm{Ca}_{x}\left(\mathrm{PO}_{4}\right)$ y formado, além da competição de $\mathrm{HCO}_{3}{ }^{-}$e $\mathrm{PO}_{4}{ }^{3-}$ por $\mathrm{Ca}^{2+}$. Dessa forma, o aumento nos valores $\mathrm{de} \mathrm{PO}_{4}{ }^{3-}$ torna-se algo atrativo do ponto de vista do reúso da AN-ROA tratada na agricultura, uma vez que o fósforo é um nutriente essencial para o crescimento das plantas e a maior parte desse nutriente, utilizado como fertilizante, provém de fontes não renováveis (Cooper et al., 2011).

A conservação de nitrogênio total no efluente tratado foi de $87 \pm 5 \%$ e de $80 \pm 8 \%$, nas bateladas com período de 12 e 6 dias (Tabela 5), respectivamente, sendo o amônio a principal forma. Assim como relatado na pesquisa de Moges et al. (2018), após a digestão anaeróbia da água negra, o aumento nos valores de $\mathrm{PO}_{4}{ }^{3-}$ e de $\mathrm{NH}_{4}{ }^{+}$ evidenciam a possibilidade de reúso dos nutrientes e os autores optaram pela posterior filtragem da água negra tratada, em filtros adsorventes, para recuperação desses nutrientes. Dessa forma, acredita-se que a alta conservação de compostos nitrogenados, na atual pesquisa, possa contribuir com a reutilização do efluente tratado para irrigação de culturas. Entretanto, caso seja necessária sua remoção, estudos realizados por de Graaff et al. (2011), no pós-tratamento por processos de nitritação - anammox de efluente de águas escuras tratada por reator UASB- de- monstraram eficientes remoções de nitrogênio total, sendo de $85 \%$ a $25^{\circ} \mathrm{C}$ e $89 \%$ a $35{ }^{\circ} \mathrm{C}$.

Tabela 5 - Valores finais dos nutrientes nas bateladas de 12 e 6 dias.

\begin{tabular}{|c|c|c|c|}
\hline Nutriente & Unidade & Bateladas de 12 dias & Bateladas de 6 dias \\
\hline NT & mg. $\mathrm{L}^{-1}$ & $251 \pm 53$ & $259 \pm 9$ \\
\hline Amônio & $\mathrm{mg} \cdot \mathrm{L}^{-1}$ & $229 \pm 61$ & $233 \pm 32$ \\
\hline Fosfato & $\mathrm{mg} \cdot \mathrm{L}^{-1}$ & $135,16 \pm 30,38$ & $142,87 \pm 26,35$ \\
\hline
\end{tabular}

A relação C: $N$ obtida do efluente $A N-R O A$ bruto foi de 2,14 nas bateladas com período de 12 dias e 1,45 nas bateladas com período de 6 dias, valores inferiores ao ideal para digestão anaeróbia, que são de 20:1 a 30:1 (DĘBOWSKI et al., 2013), sugerindo, assim, a possibilidade da introdução de uma quantia maior de ROA. Além disso, com o decorrer do tratamento, a maior remoção de matéria orgânica e a conservação de nitrogênio diminuíram essa relação, sem apresentar prejuízo ao tratamento, e, ao final das bateladas, a relação C:N obteve valores de 0,90 e 0,70 para os períodos de 12 e 6 dias, respectivamente.

\section{CONCLUSÕES}

A codigestão de água negra com resíduos orgânicos alimentares em reator UASB apresentou eficiente biodegradação da matéria orgânica e desempenho operacional satisfatório. Assim, após 81 dias de operação, foi possível a diminuição do período de tratamento das bateladas de 12 dias para 6 dias, sem prejuízo ao tratamento, obtendo remoção de $\mathrm{DQO}_{\mathrm{T}}$ de $86 \%$ e $84 \%$, respectivamente, sendo a maior parte consumida na forma de $\mathrm{DQO}_{S \mathrm{SS}}$. Além do desempenho satisfatório do reator, na remoção de material particulado, a remoção de $66 \%$ e $60 \%$ de COT nas bateladas com período de 12 e 6 dias, respectivamente, comprovou também a eficiência da codigestão na remoção de matéria orgânica. 
Além disso, durante a realização das bateladas, foi observado que o reator apresentou aumento da alcalinidade a bicarbonato $\left(\mathrm{HCO}_{3}{ }^{-}\right)$do sistema que contribuiu para manter o $\mathrm{pH}$ em faixa favorável a digestão anaeróbia, sem apresentar acúmulo de AGV.

Ao final das bateladas, foi observado que os nutrientes $\mathrm{PO}_{4}^{3-}$ e NT apresentaram eficiente conservação na AN-ROA tratada, sendo a manutenção de nitrogênio total de $87 \%$ e $80 \%$, nas bateladas com período de 12 e 6 dias, respectivamente, e a manutenção total do $P$.

Assim, devido ao potencial de recuperação de recursos das fontes segregadas do esgoto doméstico e dos resíduos orgânicos alimentares, sugere-se que novas pesquisas sejam realizadas sobre a utilização de água cinza escura (produzida em pias de cozinha) junto com a água negra, na codigestão com resíduos orgânicos alimentares, e a inclusão de pós-tratamento objetivando a remoção de patógenos e micropoluentes, a fim de permitir o reúso do efluente produzido para irrigação.

\section{AGRADECIMENTOS}

Este trabalho foi financiado pela Capes [bolsa $n^{\circ} 1513025$ ] e obteve recursos da Finep [projeto $\mathrm{n}^{\circ}$ 01.12.0112-00].

\section{CONTRIBUIÇÃO DOS AUTORES}

Conceitualização: Prado LO, Paulo PL e Boncz MÁ; Metodologia: Prado LO, Chiquito GM, Paulo PL e Boncz MÁ; Investigação: Prado LO e Chiquito GM; Redação - Primeira versão: Prado LO e Boncz MÁ; Redação - Revisão \& Edição: Prado LO e Boncz MÁ; Aquisição de Financiamento: Paulo PL; Supervisão: Boncz MÁ e Paulo PL.

\section{REFERÊNCIAS}

ASSOCIAÇÃO BRASILEIRA DE NORMAS TÉCNICAS. NBR 7229: Projeto, construção e operação de sistemas de tanques sépticos. Rio de Janeiro: ABNT, 1993. 15 p.

APHA, AWWA, WEF. Standard Methods for examination of water and wastewater. 22nd ed. Washington: American Public Health Association; 2012, $1360 \mathrm{p}$.

BRASIL. Ministério das Cidades. Secretaria Nacional de Saneamento Ambiental - SNSA. Sistema Nacional de Informações sobre Saneamento: Diagnóstico dos Serviços de Água e Esgotos - 2016. Brasília: SNSA/MCIDADES, 2018

CHERNICHARO, C. A. D. L. Reatores Anaeróbios. $2^{\mathrm{a}}$. ed. Belo Horizonte: Editora UFMG, 2007.

COOPER, J. et al. The future distribution and production of global phosphate rock reserves. Resources, Conservation and Recycling, v. 57, p. 78 - 86, 2011. https://doi.org/10.1016/j. resconrec.2011.09.009

CUNHA, J. R. et al. Simultaneous recovery of calcium phosphate granules and methane in anaerobic treatment of black water: Effect of bicarbonate and calcium fluctuations. Journal of Environmental Management, v. 216, p. 399-405, 2018. https:// doi.org/10.1016/j.jenvman.2017.09.013

DE GRAAFF, M. S. et al. Anaerobic treatment of concentrated black water in a UASB reactor at a short HRT. Water (Switzerland), v. 2, n. 1, p. 101-119, 2010. https://doi.org/10.3390/w2010101

DE GRAAFF, M. S. et al. Fate of hormones and pharmaceuticals during combined anaerobic treatment and nitrogen removal by partial nitritation-anammox in vacuum collected black water. Water research, v. 45 , n. 1, p. 375-83, jan. 2011. https://doi. org/10.1016/j.watres.2010.08.023

DĘBOWSKI, M. et al. Renewable and Sustainable Energy Reviews Algae biomass as an alternative substrate in biogas production technologies - Review. Renewable and Sustainable Energy Reviews, v. 27, p. 596-604, 2013. https://doi.org/10.1016/j. rser.2013.07.029

ELMITWALLI, T. A. et al. Anaerobic biodegradability and digestion in accumulation systems for concentrated black water and kitchen organic-wastes. Water Science and Technology, v. 53, n.8, p.16775, 2006. https://doi.org/10.2166/wst.2006.247

HERNÁNDEZ LEAL, L. et al. Characterization and anaerobic biodegradability of grey water. Desalination, v. 270, n. 1-3, p. 111-115, 2011. https://doi.org/10.1016/j.desal.2010.11.029

$\mathrm{HU}, \mathrm{M}$. et al. Constructing the ecological sanitation: A review on technology and methods. Journal of Cleaner Production, v.125, p. 1 - 21, 2016. https://doi.org/10.1016/j.jclepro.2016.03.012

INSTITUTO DE PESQUUISA ECONÔMICA APLICADA (IPEA). Plano Nacional de Resíduos Sólidos: diagnóstico dos resíduos 
urbanos, agrosilvopastoris e a questão dos catadores. Brasilia: IPEA, 2012.

JIN, Z. et al. Black water collected from the septic tank treated with a living machine system: HRT effect and microbial community structure. Chemosphere, v. 210, p. 745-752, 2018. https://doi. org/10.1016/j.chemosphere.2018.07.082

KUJAWA-ROELEVELD, K. et al. Co-digestion of concentrated black water and kitchen refuse in an accumulation system within the DESAR ( decentralized sanitation and reuse ) concept. Water Science Technology, v. 48, p. 121-128, 2003. https://doi. org/10.2166/wst.2003.0235

KUJAWA-ROELEVELD, K. et al. Performance of UASB septic tank for treatment of concentrated black water within DESAR concept. Water Science and Technology, v. 52, n. 1-2, p. 307-313, 2005. https://doi.org/10.2166/wst.2005.0532

LUOSTARINEN, S. et al. Effect of temperature on anaerobic treatment of black water in UASB-septic tank systems. Bioresource Technology, v. 98, n. 5, p. 980-986, 2007. https:// doi.org/10.1016/j.biortech.2006.04.018

LUOSTARINEN, S.; RINTALA, J. Anaerobic on-site treatment of kitchen waste in combination with black water in UASBseptic tanks at low temperatures. Bioresource technology, v. 98, n. 9, p. 1734-40, jul. 2007. https://doi.org/10.1016/j. biortech.2006.07.022

MOGES, E. M.; TODT, D.; HEISTAD, A. Treatment of SourceSeparated Blackwater: A Decentralized Strategy for Nutrient Recovery towards a Circular Economy. Water, v. 10, p. 463, 2018. https://doi.org/10.3390/w10040463

OARGA-MULEC, A. et al. Zero-discharge solution for blackwater treatment at remote tourist facilities. Journal of Cleaner Production, v. 166, p. 798-805, 2017. https://doi.org/10.1016/j. jclepro.2017.08.002

TODT, D.; JENSSEN, P. D. Particle removal in a novel sequential mechanical filter system loaded with blackwater. Water Science and Technology, v. 71, n. 9, p. 1407-1413, 2015. https://doi. org/10.2166/wst.2015.114

WENDLAND, C. et al. Anaerobic digestion of blackwater from vacuum toilets and kitchen refuse in a continuous stirred tank reactor (CSTR). Water Science and Technology, v. 55, n. 7, p. 187194, 2007. https://doi.org/10.2166/wst.2007.144.

WIELEMAKER, R. C.; WEIJMA, J.; ZEEMAN, G. Harvest to harvest: Recovering nutrients with New Sanitation systems for reuse in Urban Agriculture. Resources, Conservation and Recycling, v. 128, p. 426-437, 2018. https://doi.org/10.1016/j. resconrec.2016.09.015 